\title{
SELF-INCOMPATIBILITY IN RYEGRASS \\ VI. SELF SEED-SET AND INCOMPATIBILITY GENOTYPE IN LOLIUM PERENNE L
}

\author{
C. H. FEARON*, M. D. HAYWARD + , and M. J. LAWRENCE* \\ * Department of Genetics, University of Birmingham, Birmingham B15 2TT, England. \\ $\uparrow$ Welsh Plant Breeding Station, Plas Gogerddan, Aberystwyth SY23 3EB, Wales
}

Received 7.x.82

\section{SUMMARY}

The results from an experiment designed to investigate the possibility of an effect of incompatibility genotype on the level of pseudo-self-compatibility gave little or no evidence of such an effect among the members of three pairs of perennial ryegrass families. The plants of one of these pairs of families, however, set a little more seed than those of the others both in this and a previous experiment which indicates an effect of the genetic background on pseudo-selfcompatibility.

A revision of the classification of four plants of a previous experiment (Cornish et al., 1979) shows that the genotype of F4 is $S_{1.1} Z_{1.4}$ and that the genotype of F3, G11 and G13 is $S_{1 \cdot 2} Z_{2 \cdot 3}$.

\section{INTRODUCTION}

Though Lolium perenne is a highly self-incompatible species, it is nevertheless not completely so, for small quantities of seed are usually obtained when individual plants are self-pollinated. This behaviour is referred to as pseudo-self-compatibility in order to distinguish it from the genuine selfcompatibility that occurs when an incompatibility allele mutates to a selffertile $\left(S_{\mathrm{F}}\right)$ form. Both we (Cornish et al., 1980b; Scarrott, 1981) and others, (Jenkin, 1931; Beddows et al., 1962; Foster and Wright, 1970) have found that individual plants vary with respect to their pseudo-selfcompatibility and that both genetical and environmental factors appear to be involved. Hitherto, it has not been possible to find out whether there is any relationship between the level of pseudo-self-compatibility and incompatibility genotype in perennial ryegrass because the genetical control of incompatibility in this species was not known. Having shown recently that incompatibility in this species is controlled, as in other grasses, by a pair of multi-allelic genes with gametophytic action in the pollen (Cornish et al., 1979), it is now possible to find out whether different $S$ and $Z$ alleles, either individually or in combination, vary in their ability to prevent self-pollination. The experiment described in this paper was designed with this question in mind. We also give some further information about the genotype of the anomalous plant F4 mentioned by Cornish et al. (1980b) and a revision of the genotypes of three other members of the same cross.

\section{Materials ANd Methods}

The plants used in the experiment were clonal replicates of those whose incompatibility genotype had been found by Cornish et al., (1979). In all, 
163 plants were self-pollinated, 61 from reciprocal families D and E, 34 from families $F$ and $G$ and 68 plants from families $H$ and $I$. While all, or nearly all, of the plants of families D, E, H and I were used in the present experiment, only half of the total number from families $F$ and $G$ were available, as the remainder were required for another purpose. The position of each plant on the glasshouse bench was decided at random and the experiment was carried out during the natural flowering season of the species during the summer of 1980. Further details of the experiment are given in Scarrott (1981).

\section{Results}

\section{(i) Families $H$ and $I$}

The average percentage seed-set obtained by self-pollinating plants from the reciprocal families $\mathrm{H}$ and $\mathrm{I}$ are shown in table 1. One plant (H22) gave a seed-set of 17.66 per cent. Since this is much higher than the seed-set obtained by selfing the same plant on a previous occasion $(0.26$ per cent $)$ and is also much higher than the seed-set of any other plant in the experiment, this result is almost certainly due to contamination by compatible pollen prior to bagging up. The seed-set of this plant, which was of genotype $S_{1 \cdot 3} Z_{1 \cdot 4}$, has therefore been omitted from the data shown in table 1 .

TABLE 1

Families $H$ and I. Mean percentage self seed-set (upper) and number of plants (lower) for each class

\begin{tabular}{cccccc}
\hline Genotype & $S_{1} S_{3}$ & $S_{1} S_{4}$ & $S_{2} S_{3}$ & $S_{2} S_{4}$ & Means \\
\hline \multirow{2}{*}{$Z_{1} Z_{3}$} & 0.08 & 0.14 & $0 \cdot 10$ & 0.23 & 0.14 \\
& 4 & 10 & 6 & 6 & 26 \\
$Z_{1} Z_{4}$ & 1.80 & 0.88 & & 0.53 & $1 \cdot 02$ \\
& 3 & 3 & & 4 & 10 \\
$Z_{2} Z_{3}$ & 0.63 & 0.20 & $0 \cdot 12$ & 0.07 & 0.32 \\
& {$[6]$} & 4 & 3 & 3 & 16 \\
$Z_{2} Z_{4}$ & $0 \cdot 19$ & & 0.27 & 0.01 & 0.16 \\
& 4 & & 6 & 5 & 15 \\
\hline \multirow{2}{*}{ Means } & 0.60 & 0.28 & 0.17 & 0.20 & 0.32 \\
& 17 & 17 & 15 & 18 & 67 \\
\hline
\end{tabular}

The analysis of variance of these data is slightly complicated by the fact that the number of plants in each class is not the same and, in particular, that two of these classes $\left(S_{1 \cdot 4} Z_{2 \cdot 4}\right.$ and $\left.S_{2 \cdot 3} Z_{1 \cdot 4}\right)$ are empty. The missing values were estimated by a procedure given by Snedecor and Cochran (1967; section 11.9) and the problem of unequal class numbers was overcome by carrying out an approximate two-way analysis of variance of the data with the class numbers within each row made equal (Snedecor and Cochran, loc. cit., section 16.3). The original data were transformed from the percentage to the angular scale before analysis and the degrees of freedom of the Between Genotypes and the Interaction items reduced by 
two because two missing values have been estimated from the data (table 2).

TABLE 2

Families $H$ and I. Analysis of variance of self seed-set. Data transformed from percentages to angles before analysis. The theoretical error is $820.7 / N$, where $N$ is the harmonic mean of the estimated numbers of florets per unit $\left(^{* * *}, P<0.001\right)$

\begin{tabular}{llc}
\hline \multicolumn{1}{c}{ Item } & d.f. & M.S. \\
\hline Between genotypes & 13 & $8 \cdot 861$ \\
Between $S_{i} S_{j}$ & {$[3]$} & $2 \cdot 415$ \\
Between $Z_{i} Z_{j}$ & {$[3]$} & $29 \cdot 116^{* * *}$ \\
Interaction & {$[7]$} & $2 \cdot 944$ \\
Within Genotypes & 53 & $4 \cdot 645^{* * *}$ \\
Theoretical Error & $\infty$ & $0 \cdot 335$ \\
\hline
\end{tabular}

There is no evidence in these data of any effect of $S$ genotype on self seed-set either on average or in combination with $Z$ genotype; the latter, on the other hand, does appear to have a significant effect. The possibility that this is due to the approximations made in the analysis or estimation of missing values is countered by carrying out a conventional one-way analysis of these data in which differences between plants with respect to their $S$-genotype are ignored, for the Between $Z_{i} Z_{j}$ item of this simpler analysis is still highly significant $(P=0.01-0.001)$. The effect of $Z$ genotype on self seed-set appears, therefore, to be genuine. Inspection of the data shows that despite the exclusion of plant $\mathrm{H} 22$, the remaining plants of the same genotype $\left(Z_{1} Z_{4}\right)$ set, on average, slightly more seed on self-pollination than those of other $Z$-genotypes (table 3 ). Lastly, we note that, as in previous experiments (Cornish et al., 1980b), the Within Genotypes M.S. is much larger than the binomial error variance.

TABLE 3

Mean self seed-set of plants of the indicated $Z$ genotype

\begin{tabular}{ccc}
\hline Genotype & Mean seed-set $(\%)$ & Number of plants \\
\hline$Z_{1} Z_{3}$ & 0.14 & 26 \\
$Z_{1} Z_{4}$ & $1 \cdot 02$ & 10 \\
$Z_{2} Z_{3}$ & $0 \cdot 32$ & 16 \\
$Z_{2} Z_{4}$ & 0.16 & 15 \\
\hline
\end{tabular}

\section{(ii) Families $D$ and $E$}

The average self seed-set of the plants of reciprocal families D and E are shown in table 4. Though in this pair of families each of the sixteen genotypic classes is represented by at least one plant, the data are in other respects similar to those of families $\mathrm{H}$ and $\mathrm{I}$ and have been analysed in the same way accordingly (table 5 ). The analysis of variance shows that there is no evidence of any effect of $S$ or $Z$ genotype on self seed-set in these data. 
TABLE 4

Families $D$ and E. Mean percentage self seed-set (upper) and number of plants (lower) for each class. Note that while the assignation of plants to classes is consistent over the reciprocal families of a cross, the designation of genotypes over different crosses is arbitrary

\begin{tabular}{cccccc}
\hline Genotype & $S_{1} S_{3}$ & $S_{1} S_{4}$ & $S_{2} S_{3}$ & $S_{2} S_{4}$ & Means \\
\cline { 2 - 6 }$Z_{1} Z_{3}$ & 1.21 & 0.09 & 0.05 & $0 \cdot 17$ & 0.52 \\
& 4 & 1 & 2 & 4 & 11 \\
$Z_{1} Z_{4}$ & $0 \cdot 11$ & 0.09 & 0.23 & 0.39 & 0.23 \\
& 4 & 2 & 8 & 5 & 19 \\
$Z_{2} Z_{3}$ & $0 \cdot 14$ & 0.12 & 0.83 & $0 \cdot 22$ & $0 \cdot 27$ \\
& 4 & 3 & 2 & 4 & 13 \\
$Z_{2} Z_{4}$ & 0.21 & 0.07 & 0.20 & 0.17 & 0.17 \\
& 5 & 4 & 6 & 3 & 18 \\
\hline \multirow{2}{*}{ Means } & 0.40 & 0.09 & 0.27 & 0.25 & 0.27 \\
& 17 & 10 & 18 & 16 & 61 \\
\hline
\end{tabular}

TABLE 5

Families D and E. Analysis of variance of self seed-set. Other details as for table 2

\begin{tabular}{lll}
\hline \multicolumn{1}{c}{ Item } & d.f. & M.S. \\
\hline Between genotypes & 15 & 3.009 \\
Between $S_{i} S_{j}$ & {$[3]$} & $3 \cdot 281$ \\
Between $Z_{i} Z_{j}$ & {$[3]$} & 1.316 \\
Interaction & {$[9]$} & 3.482 \\
Within genotypes & 45 & $4 \cdot 024^{* * *}$ \\
Theoretical error & $\infty$ & $0 \cdot 271$ \\
\hline
\end{tabular}

\section{(iii) Families $F$ and $G$}

The data from this pair of families are too incomplete to allow even an approximate two-way analysis of variance, for only half of the classes have any entries (table 6). However, one-way analyses of variance of differences between $S$-genotype on the one hand and between $Z$-genotype on the other failed to show any effect of either on self seed-set (table 7).

\section{(iv) The three crosses combined}

A summary of the data obtained from each of the three pairs of families involved in this experiment shows that average self seed-set is a little higher in $F$ and $G$ than it is in the other families (table 8(a)). Furthermore, this small difference is highly significant (table $8(b)$ ). Since a similar result was obtained from a previous experiment (Cornish et al., 1980b), it is likely that this difference is genetic rather than environmental.

\section{(v) The anomalous plant, F4}

Cornish et al., (loc. cit.) obtained a 19.20 per cent seed-set from the cross $\mathrm{F} 4 \times \mathrm{F} 36$ which was predicted to be incompatible, since both plants 
TABLE 6

Families $F$ and $G$. Mean percentage self seed-set (upper) and number of plants (lower) for each class. Other details as for table 4 (the anomalous plant F4 was excluded from this experiment-see text)

\begin{tabular}{cccccc}
\hline Genotype & $S_{1} S_{1}$ & $S_{1} S_{3}$ & $S_{1} S_{2}$ & $S_{2} S_{3}$ & Means \\
\hline$Z_{1} Z_{3}$ & & 1.17 & 0.26 & & 0.83 \\
& & 10 & 6 & & 16 \\
$Z_{1} Z_{4}$ & & 0.72 & & & 0.72 \\
$Z_{2} Z_{3}$ & & 3 & & & 3 \\
& & & 0.44 & & 0.44 \\
$Z_{2} Z_{4}$ & 1.61 & 0.94 & 3.06 & 1.36 & 1.33 \\
& 2 & 6 & 1 & 3 & 12 \\
\hline \multirow{2}{*}{ Means } & 1.61 & 1.03 & 0.60 & 1.36 & 0.96 \\
& 2 & 19 & 10 & 3 & 34 \\
\hline
\end{tabular}

TABLE 7

Families $F$ and $G$. (a) Analysis of variance of differences between $\boldsymbol{S}_{i} \boldsymbol{S}_{j}$ genotypes. (b) Analysis of variance of differences between $Z_{i} Z_{j}$ genotypes. Other details as for tables 2 and 5

(a)

\begin{tabular}{lcc}
\hline \multicolumn{1}{c}{ Item } & d.f. & M.S. \\
\hline Between $\boldsymbol{S}_{i} \boldsymbol{S}_{\boldsymbol{i}}$ & 3 & $11 \cdot 192$ \\
Within genotypes & 30 & $8 \cdot 835^{* * *}$ \\
Theoretical error & $\infty$ & $0 \cdot 357$ \\
\hline
\end{tabular}

(b)

\begin{tabular}{lrl}
\hline \multicolumn{1}{c}{ Item } & d.f. & \multicolumn{1}{c}{ M.S. } \\
\hline Between $Z_{i} Z_{i}$ & 3 & 6.903 \\
Within genotypes & 30 & $9 \cdot 264^{* * * *}$ \\
Theoretical error & $\infty$ & $0 \cdot 357$ \\
\hline
\end{tabular}

had been classified originally as being of genotype $S_{1 \cdot 3} Z_{1 \cdot 4}$. The reciprocal cross gave only a 0.17 per cent seed-set and both plants when selfed gave either little or no seed (F4, 0 per cent; F36, 0.89 per cent). It was suggested that F4 had been originally misclassified and that its genotype was in fact $S_{1.1} Z_{1 \cdot 4}$; or that a mistake had been made during clonal replication of the stock plant subsequent to its classification; or possibly, that the cross F $4 \times$ F36 had been contaminated by pollen from another plant.

This uncertainty has now been resolved. Thus when F4 was crossed with each of four plants from the same family that had been classified as being of genotype $S_{1 \cdot 1} Z_{1 \cdot 4}$, incompatible reactions were always observed irrespective of the direction of the cross. Again, when used as a female parent in crosses with another plant that had been classified as $S_{1 \cdot 3} Z_{1 \cdot 4}$, a half-compatible reaction was obtained. The cross $\mathrm{F} 4 \times \mathrm{F} 36$ was also 
TABLE 8

The three crosses combined. (a) Summary of self seed-set averaged over all the plants of each of the three pairs of families. The data in brackets are estimates of the seed-set obtained from both selfs and incompatible crosses involving plants from the same crosses that were obtained from a previous experiment (Cornish 1979; Cornish et al., 1980b). Subscripts indicate the number of plants (and/or crosses) on which the seed-set estimates are based. (b) Analysis of variance of the combined seed-set data of the present experiment

(a)

\begin{tabular}{cc}
\hline Families & Mean self seed-set $(\%)$ \\
\hline $\mathrm{D}+\mathrm{E}$ & $0 \cdot 27_{61}\left(0 \cdot 08_{56}\right)$ \\
$\mathrm{F}+\mathrm{G}$ & $0.96_{34}\left(0 \cdot 75_{39}\right)$ \\
$\mathrm{H}+\mathrm{I}$ & $0.32_{68}\left(0 \cdot 10_{20}\right)$ \\
\hline
\end{tabular}

(b)

\begin{tabular}{lcc}
\hline \multicolumn{1}{c}{ Item } & d.f. & M.S. \\
\hline Between families & 2 & $80 \cdot 722^{* * *}$ \\
Within families & 159 & $5 \cdot 387^{* * *}$ \\
Theoretical error & $\infty$ & $0 \cdot 315$ \\
\hline
\end{tabular}

repeated with the same outcome. Thus it is now clear that the genotype of F4 is in fact $S_{1 \cdot 1} Z_{1 \cdot 4}$, and not $S_{1 \cdot 3} Z_{1 \cdot 4}$ as was originally reported.

Apart from the need to resolve this uncertainty for obvious reasons, plant F4 has been used as a parent in another experiment concerning the genetics of metrical characters, details of which will be published in due course.

\section{(vi) A revision of the classification of plants F3, G11 and $G 13$}

Plants F3, G11 and G13, which, as it happens, are members of the same pair of families as F4, were originally classified as being of genotype $S_{1 \cdot 3} Z_{2 \cdot 3}$ (Cornish et. al., 1979). The cross which gave rise to families $\mathrm{F}$ and $\mathrm{G}$ was of the type $S_{1 \cdot 2} Z_{1 \cdot 2} \times S_{1 \cdot 3} Z_{3 \cdot 4}$, that is, the parents had an $S$-allele in common. Although plants of $S_{1 \cdot 2} Z_{2 \cdot 3}$ genotype are expected in the progeny of such a cross none were apparently found (see table 3 of Cornish et al., loc. cit.). However, the pattern of pollinations expected when plants of genotype $S_{1.3} Z_{2.3}$ and $S_{1.2} Z_{2 \cdot 3}$ are crossed to each of the other genotypes expected in these families are very similar, it being necessary to distinguish between half- and three-quarters compatible reactions in order to assign a plant to one or other of these genotypic classes, irrespective of whether such plants are used as males or females.

Recently, in connection with another piece of work, plants F3, G11 and G13 were pollinated by plants of genotype $S_{1 \cdot 2} Z_{3 \cdot 3}, S_{1 \cdot 1} Z_{3 \cdot 3}$ and $S_{2 \cdot 2} Z_{3 \cdot 3}$, with which they were expected to be half-compatible, incompatible and fully compatible, respectively, if their original classification had been correct. Instead, all of these pollinations were incompatible. It is thus now 
clear that the genotype of plants F3, G11 and G13 is, in fact, $S_{1.2} Z_{2.3}$ and not $S_{1 \cdot 3} Z_{2 \cdot 3}$ as originally reported.

Although the revision of the genotype of plant F4 has no effect on the estimate of linkage between $S$ and $P G I-2$ in these families (Cornish et al., $1980 a$ ), the revision of the genotype of the others does change this estimate. Thus the revised estimate for the cross is $\hat{p}_{\mathrm{FG}}=0.2121 \pm 0.0503$ (instead of $\hat{p}_{\mathrm{FG}}=0 \cdot 1970 \pm 0.0490$ ) and the revised overall joint estimate of the linkage between $S$ and $P G I-2$ obtained by pooling the data over the three crosses is $\hat{p}=0.1694 \pm 0.0276$ (instead of $\hat{p}=0.1583 \pm 0.0252$ ) and the new heterogeneity $\chi_{(2)}^{2}=4.867, P=0.10-0.05$ (instead of $\chi_{(2)}^{2}=4.076$, $P=0.2-0 \cdot 1)$.

\section{Discussion}

Taken as a whole, the results of this experiment confirm once again that perennial ryegrass is a highly, though not completely, self-incompatible species. Yet because the total number of florets borne by a single well-grown individual is large, even a slight expression of pseudo-self-compatibility can yield a small, though useful, amount of self-seed.

As to the cause of pseudo-self-compatibility in these families, there is little evidence in these data that different $S$ and $Z$ alleles differ in their ability to prevent self-pollination. Though the size of these families is, perhaps, smaller than is desirable, with one exception, the Between Genotypes mean squares of the analyses of variance (tables 2, 5 and 7) are smaller than their respective Within Genotype mean squares. Thus there is little to encourage the belief that had these families been larger, we would have found the effect that we were seeking. Furthermore, the evidence of some effect of $Z$-genotype on self seed-set in families $\mathrm{H}$ and $I$ is by no means as convincing as appears at first sight. First, the effect appears to be confined to one genotype, $Z_{1} Z_{4}$, the seed-set of plants of the other three genotypes being similar to the overall average for the pair of families (tables 1 and 3 ). Second, the $Z_{1} Z_{4}$ class is the least well represented of the four, containing only ten plants. Last, the relatively high average seed-set of the plants of this class depends on the high scores of just two plants whose seed-set was 4.81 and $2 \cdot 18$ per cent respectively; the average seed-set of the remaining eight plants is, at 0.40 per cent, comfortably within the range of the average values of the plants of the other three classes and hence similar to the overall average for the pair of families. Taken as a whole, therefore, there is no evidence of any effect of incompatibility genotype on pseudo-self-compatibility in two of our pairs of families and in the third, where there is, the evidence effectively rests on two plants only.

On the other hand, there is some evidence of such an effect in other species. Thus Lundqvist (1958) found that the level of self-fertility was higher for double than for single heterozygotes in inbred families of rye; and that the $S$ and $Z$ alleles segregating in an inbred family of Festuca pratensis differed in their efficiency in preventing self-pollination as assessed by looking at the behaviour of pollen on the stigma (Lundqvist 1964). There is also evidence of this kind, of course, from species with one-locus systems of self-incompatibility. Thus East (1934) found that different $S$ homozygotes of Nicotiana sanderae varied in their levels of pseudo-self- 
compatibility; and Williams (1951), that Trifolium hybridum plants of two of the four incompatibility classes that were present in each of two F1 families set more self seed than those of the other two classes. There is little doubt, therefore, that the incompatibility genotype of an individual can affect its pseudo-self-compatibility, though it is perhaps significant that much of this evidence comes from material that has been inbred; that is, the phenomenon may not be expressed in non-inbred plants of the species in question.

While there may be little evidence of any effect of incompatibility genotype on pseudo-self-compatibility in perennial ryegrass, the slightly higher seed-set obtained on each of two occasions from the plants of families $\mathrm{F}$ and $\mathrm{G}$ fairly clearly indicates some effect of genetic background. This result is similar to those of Jenkin (1931), Beddows et al., (1962), Hayward and Breese (1966) and Foster and Wright (1970), all of whom showed that there were consistent differences for self seed-set between ryegrass genotypes over replicates and in two of these investigations, over seasons also. Furthermore the studies of Utz and Oettler (1978) and Jones and Jenabzadeh (1981) clearly show that sufficient genetic variation in self fertility exists for selection to be effective, allowing the establishment of inbred lines. These observations, together with those from other species (e.g. Mather, 1943 with Petunia hybrids; Atwood, 1942 with Trifolium repens; Martin, 1967 with Lycopersicon spp.; and Denward, 1963a, b, c, $d$ with Trifolium pratense), confirm the view that an efficient self-incompatibility system depends on the adjustment by natural selection of both the incompatibility genes themselves and the genetical milieu in which their effects are expressed.

Acknowledgement. We wish to acknowledge the award of a Science Research Council CASE studentship to C. H. Fearon (née Scarrott) which enabled the work described in this paper to be carried out.

\section{REFERENCES}

ATWOOD, S. S. 1942. Genetics of pseudo-self-compatibility and its relation to cross-incompatibility in Trifolium repens. J. Agri, Res., 64, 699-709.

BEDDOWS, A. R., BREESE, E. L. AND LEWIS, BETTY. 1962. The genetic assessment of heterozygous breeding material by means of a diallel cross. I. Description of parents, self- and cross-fertility and early seedling vigour. Heredity, 17, 501-512.

CORNISH, M. A. 1979. The Genetics of Self-Incompatibility in Lolium perenne Ph.D. thesis. University of Birmingham.

CORNISH, M. A., HAYWARD, M. D. AND LAWRENCE. M. J. 1979. Self-incompatibility in ryegrass. I. Genetic control in diploid Lolium perenne L. Heredity, 43, 95-106.

CORNISH. M. A., HAYWARD. M. D. AND LAWRENCE, M. J. $1980 a$. Self-incompatibility in ryegrass. III. The joint segregation of $S$ and $P G I-2$ in Lolium perenne L. Heredity, 44, 55-62.

CORNISH, M. A., HAYWARD, M. D. AND LAWRENCE, M. J. 1980b. Self-incompatibility in ryegrass. IV. Seed-set in diploid Lolium perenne L. Heredity, 44, 333-340.

DENWARD, T. 1963a. The function of the incompatibility alleles in red clover (Trifolium pratense L.). I. The effect of grafting upon self-fertility. Hereditas, 49, 189-202.

DENWARD, T. $1963 \mathrm{~b}$. The function of the incompatibility alleles in red clover (Trifolium pratense L.). II. Results of crosses within inbred families. Hereditas, 49, 203-236.

DENWARD, T. $1963 \mathrm{c}$. The function of the incompatibility alleles in red clover (Trifolium pratense L.). III. Changes in the $S$-specificity. Hereditas, 49, 285-329.

DENWARD, T. $1963 d$. The function of the incompatibility alleles in red clover (Trifolium pratense L.). IV. Resume. Hereditas, 49, 330-334. 
EAST, E. M. 1934. Norms of pollen-tube growth in incompatible matings of self-sterile plants. Proc. Nat. Acad. Sci., 20, 225-230.

FOSTER, C. A. AND WRIGHT, C. E. 1970. Variation in the expression of self-fertility in Lolium perenne. Euphytica, 19, 61-70.

HAYWARD, M. J. AND BREESE, E. L. 1966. The genetic organisation of natural populations of Lolium perenne. I. Seed and seedling characters. Heredity, 21, 287-304.

JENKIN, T. J. 1931. Self-fertility in perennial ryegrass (Lolium perenne L.). Welsh Pl. Breed. Stn. Bull. Series H No. 12, 100-119.

JONES, R. N. AND JENABZADEH, P. 1981. Variation in self-fertility, flowering time and inflorescence production in inbred Lolium perenne L. J. Agric. Sci., Camb., 96, 521-537.

LUNDQVIST, A. 1958. Self-incompatibility in rye. IV. Factors related to self-seeding. Hereditas, 44, 193-256.

LUNDQVIST, A. 1964. The nature of the two-loci incompatibility system in grasses. IV. Interaction between the loci in relation to pseudo-compatibility in Festruca pratensis Huds. Hereditas, 52, 221-234.

MARTIN, F. W. 1967. The genetic control of unilateral incompatibility between two tomato species. Genetics, 56, 391-398.

MATHER, K. 1943. Specific differences in Petunia. I. Incompatibility J. Genetics, 45, 215-235.

SCARROTT, C. H. 1981. Self-Incompatibility in Diploid and Tetraploid Lolium species. Ph.D. Thesis. University of Birmingham.

SNEDECOR, G. W. AND COCHRAN, W. G. 1967. Statistical Methods. Iowa State University Press, 6th Edn.

WILLIAMS, w. 1951. Genetics of incompatibility in Alsike clover, Trifolium hybridum. Heredity, 5, 51-73.

UTZ, H. F. AND OETTLER, G. 1978. Performance of inbred lines and their top crosses in perennial ryegrass (Lolium perenne L.) Z. Pflanzenzüchtg, 80, 223-229. 